\title{
A Daphnia Parasite (Caullerya mesnili) Constitutes a New Member of the Ichthyosporea, a Group of Protists near the Animal-Fungi Divergence
}

\author{
JENNIFER N. LOHR, ${ }^{1}$ CHRISTIAN LAFORSCH, ${ }^{1}$ HENRIKE KOERNER ${ }^{2}$ and JUSTYNA WOLINSKA \\ Department Biologie II, Ludwig-Maximilians-Universität München, Evolutionsökologie, Großhaderner Street 2, \\ D-82152 Planegg-Martinsried, Germany
}

\begin{abstract}
Caullerya mesnili is a protozoan endoparasite in the gut epithelium of Daphnia, which causes regular epidemics in lakes throughout Europe. Its classification has remained unchanged for over a century, leaving it placed with the Haplosporidia, despite speculation that this position is incorrect. The difficulty in classifying C. mesnili stems from its few known morphological and ecological characteristics, as well as a lack of genetic markers. Here we sequenced the nuclear small subunit (SSU) and internal transcribed spacer rDNA regions of $C$. mesnili samples from 10 locations. Based on sequence similarities, we suggest the re-classification of $C$. mesnili to the Ichthyosporea, a class of protists near the animal-fungi divergence. We report average intragenomic variation of $0.75 \%$ and $2.27 \%$ in the SSU and internal transcribed spacer regions, respectively. From electron micrographs and light microscopy of histological sections we determined that $C$. mesnili spores grow within the intestinal epithelium where they establish themselves intercellularly. In addition, we confirmed previous accounts regarding the high virulence of this parasite. Caullerya mesnili reduces host lifespan, the number of clutches, and the total number of offspring. This high selection pressure placed on hosts supports the importance of C. mesnili as a model parasite for the study of host-parasite biology in permanent lakes.
\end{abstract}

Key Words. Basal metazoan, host-parasite, Ichthyophonida, opisthokont, small subunit ribosomal DNA.

$\mathrm{T}$ HE Ichthyosporea, also known as the Mesomycetozoa (Mendoza, Taylor, and Ajello 2002), is a class of protozoan parasites near the animal-fungi dichotomy, owing much of their described diversity to modern sequencing techniques (e.g. Cafaro 2005). The group was first suggested by Ragan et al. (1996) and at that time labeled the DRIP clade after its four founding members, Dermocystidum, rosette agent, Ichthyophonus, and Psorospermium. Cavalier-Smith (1998) further subdivided the group into two orders based on sequence data: the Ichthyophonida (Ichthyophonae in Mendoza et al. 2002), characterized morphologically by an amoeboid stage in the life cycle and the Dermocystida (Rhinosporideacae in Mendoza et al. 2002) with a flagellate stage. However, the majority of the Ichthyosporea remain poorly described. Even within these two principle clades, the Ichthyophonida and the Dermocystida, many species lack sufficient study to confirm or refute the existence of an amoeboid or flagellate stage. The few ecological and morphological features that unify the Ichthyosporea are a parasitic lifestyle, as these organisms often infect either freshwater or marine hosts (although also mammals and birds are infected), and the formation of unicellular spores (Mendoza et al. 2002). Thus, due to their microscopic size and lack of defining morphological characteristics, most ichthyosporeans were originally placed with either the fungi or the Haplosporidia, and the class Ichthyosporea itself was not founded until the introduction of a molecular phylogeny based on small subunit ribosomal DNA (SSU rDNA) sequences.

Caullerya mesnili (Chatton 1907) is a common parasite of the crustacean waterflea Daphnia. It causes regular epidemics in large permanent lakes throughout Europe, reaching prevalences up to $40 \%$ (Wolinska et al. 2007a). Its classification has remained unchanged for over a century, leaving it placed with the Haplosporidia (Chatton 1907; Green 1974), despite speculation that this position is incorrect (Bittner, Ebert, and Rothhaupt 2002;

Corresponding Author: J. Lohr, Unité d'Ecologie et d'Evolution, Département de Biologie, Université de Fribourg, Chemin du Museé 10, 1700 Fribourg, Switzerland-Telephone number: +4102630088 57; Fax number: +49 089218074 204; e-mail: jennifer.lohr@unifr.ch ${ }^{1}$ Contributed equally.

${ }^{2}$ Present Address: Department Neuroimmunologie, Institut für Multiple Sklerose Forschung, Georg-August Universität, Waldweg 33, 37073 Göttingen, Germany.
Ebert 2005). The difficulty in its classification stems largely from a lack of detailed morphological study and genetic markers for the parasite. Regarding its life cycle, C. mesnili is known to form spore clusters in the gut epithelium of Daphnia, each cluster containing 8-20 spores, $10 \times 8 \mu \mathrm{m}$ in size (Bittner et al. 2002; Green 1974). The infection is spread horizontally by a waterborne stage directly from Daphnia to Daphnia, with new infections becoming visible from 8 to 12 days post-exposure (Bittner et al. 2002). Both lab and field studies have shown $C$. mesnili to be a virulent parasite: in the Daphnia longispina hybrid complex, egg numbers were reduced by about $95 \%$ in infected hosts (Wolinska et al. 2006; 2007a). In addition, Daphnia galeata infected in the lab with $C$. mesnili reach a smaller adult size and die earlier than healthy individuals (Lass and Bittner 2002; Wolinska et al. 2006).

Caullerya mesnili is an important addition to the current Daphnia-microparasite models available (reviewed in Ebert 2005), as it is a common parasite of large permanent lakes. Thus, C. mesnili offers a system to study host-parasite coevolution in year-round habits where the evolutionary dynamics are very different from seasonal ponds. In such permanent lakes Daphnia's clonal reproduction is rarely interrupted by the sexual phase (Keller et al. 2007). This contrasts with temporary ponds where sexually produced eggs, which have been lying dormant, may enter the population at a later time and disrupt coevolutionary cycles (Little and Ebert 2001, 2004). In addition, as C. mesnili belongs to a basal clade of protists near the animal-fungi divergence, knowledge of its phylogenetic position will clarify our understanding of basal metazoan evolution. Here we suggest the reclassification of C. mesnili, document its morphology and ultrastucture, and finally we explore several aspects of $C$. mesnili's transmission.

\section{MATERIALS AND METHODS}

Specimen collection for genetic analyses. In addition to the C. mesnili strain used in the experimental and morphological analyses, which was isolated from lake Greifensee in Switzerland (latitude $47^{\circ} 37^{\prime} \mathrm{N}$, longitude $8^{\circ} 68^{\prime} \mathrm{E}$ ), parasites from nine other locations were identified using genetic markers (Table 1). The infected Daphnia were from zooplankton samples collected in 2004/2005 from eight reservoirs in the Czech Republic (i.e. Brno $49^{\circ} 14^{\prime} \mathrm{N}, 16^{\circ} 31^{\prime} \mathrm{W}$; Ŕímov $48^{\circ} 50^{\prime} \mathrm{N}, 1^{\circ} 30^{\prime} \mathrm{W}$; Seč $49^{\circ}$ $50^{\prime} \mathrm{N}, \quad 15^{\circ} 39^{\prime} \mathrm{W} ; \quad$ Stanovice $50^{\circ} 11^{\prime} \mathrm{N}, 12^{\circ} 53^{\prime} \mathrm{W}$; Trnávka $49^{\circ} 31^{\prime} \mathrm{N}, 15^{\circ} 13^{\prime} \mathrm{W}$; Vir $49^{\circ} 34^{\prime} \mathrm{N}, 16^{\circ} 19^{\prime} \mathrm{W}$; Vranov $48^{\circ} 54^{\prime} \mathrm{N}$, $15^{\circ} 49^{\prime} \mathrm{W}$; Želivka $49^{\circ} 43^{\prime} \mathrm{N}, 15^{\circ} 06^{\prime} \mathrm{W}$; see Seda et al. 2007 for 
Table 1. List of molecularly identified Caullerya mesnili isolates, their respective sampling locations and GenBank accession numbers.

\begin{tabular}{|c|c|c|c|c|}
\hline \multirow[t]{2}{*}{ Location } & \multirow[t]{2}{*}{ Season } & \multirow[t]{2}{*}{ Number of isolates ${ }^{a}$} & \multicolumn{2}{|c|}{ Accession Number (GenBank) ${ }^{\mathrm{b}}$} \\
\hline & & & SSU & ITS \\
\hline Ammersee, Germany & September 2008 & 2 & GU123071 & GU123079-84 \\
\hline Brno, Czech Republic & October 2005 & 3 & $G U 123048-53$ & GU123085-90 \\
\hline Greifensee, Switzerland & September 2006 & $1^{\mathrm{c}}$ & GU123072, GU123054-58 & GU123091-96 \\
\hline Rimov, Czech Republic & October 2005 & 2 & GU123073 & GU123097-102 \\
\hline Seč, Czech Republic & October 2005 & 2 & $G U 123059-64$ & GU123103-108 \\
\hline Stanovice, Czech Republic & October 2005 & 2 & GU123074 & GU123109-114 \\
\hline Trnávka, Czech Republic & October 2005 & 2 & GU123075 & GU123115-120 \\
\hline Vir, Czech Republic & October 2005 & 2 & GU123076 & $G U 123121-126$ \\
\hline Vranov, Czech Republic & October 2005 & 2 & GU123077 & $G U 123127-132$ \\
\hline Želivka, Czech Republic & October 2004/2005 & 2 & GU123078, GU123065-70 & GU123133-138 \\
\hline
\end{tabular}

${ }^{\mathrm{a}}$ From each location, at least one isolate was completely sequenced in the SSU and ITS regions. For all other isolates, partial sequence information was sufficient to unambiguously identify the parasite taxon.

${ }^{\mathrm{b}}$ The GenBank accession numbers are provided for the isolates that were completely sequenced for the SSU and ITS regions. Italicized entries indicate that the PCR product was cloned (as opposed to direct sequencing).

${ }^{\mathrm{c}}$ The parasite strain was isolated from lake Greifensee and kept in a Daphnia galeata laboratory clone obtained from the same lake.

All parasites were isolated from hosts that belong to the Daphnia longispina hybrid complex.

SSU, small subunit; PCR, polymerase chain reaction.

detailed sampling procedures and a description of the study site) and in 2008 from one natural lake in Germany (Ammersee $48^{\circ} 02^{\prime} \mathrm{N}, 11^{\circ} 12^{\prime} \mathrm{W}$ ). All infected hosts belonged to the $D$. longispina hybrid complex, as confirmed by morphology (i.e. D. galeata, Daphnia hyalina, Daphnia cucullata, and their respective hybrids; Petrusek et al. 2008).

Specimen collection for experimental and morphological analyses. The parasite strain used for laboratory studies was collected in 2006 from a natural lake in Switzerland (Greifensee). The parasite was maintained in a $D$. galeata genotype collected from the same lake by adding newborns from uninfected stock cultures at approximately 2-week intervals (as described in Wolinska et al. 2006). The daphniids were reared with an unlimited food supply of the unicellular green algae Scenedesmus obliquus in artificial medium (for details see Jeschke and Tollrian 2000). Both host and parasite were kept in climate chambers at $20 \pm 1{ }^{\circ} \mathrm{C}$ with a summer photoperiod of 16:8 light-dark.

Molecular data. Genomic DNA extraction, polymerase chain reaction (PCR) amplification, cloning, and sequencing. Genomic DNA was isolated by overnight incubation of single infected Daphnia with $100 \mu \mathrm{g} / \mathrm{ml}$ proteinase K (Merck KGaA, Darmstadt, Germany) and $0.1 \%$ SDS in proteinase $\mathrm{K}$ buffer $(10 \mathrm{mM}$ Tris/HCl
pH 8.0, $100 \mathrm{mM} \mathrm{NaCl}, 25 \mathrm{mM}$ EDTA $\mathrm{pH} 8.0$ ) at $55^{\circ} \mathrm{C}$. After inactivation of proteinase $\mathrm{K}$ for $12 \mathrm{~min}$ at $95^{\circ} \mathrm{C}$, DNA was precipitated using isopropanol and then dissolved in $50 \mu 1$ of sterile water. For the initial determination of nucleotide sequences of the rDNA regions, DNA of the parasite from Greifensee, Switzerland (extracted from lab-infected D. galeata) was amplified using universal primers for the eukaryotic SSU rDNA region (Table 2). Polymerase chain reaction was carried out with 2-3 $\mu 1$ of genomic DNA, $0.05 \mathrm{U} / \mu 1$ DreamTaq DNA polymerase, $0.02 \mathrm{U} / \mu 1 \mathrm{Pfu}$ DNA polymerase, $1 \times$ DreamTaq buffer, $0.25 \mathrm{mM}$ dNTPs (all from Fermentas GmbH, St. Leon-Rot, Germany), and $0.5 \mu \mathrm{M}$ of each primer (Metabion, Martinsried, Germany). Polymerase chain reaction amplicons of the correct size were gel eluted using the QIAquick gel extraction kit (Qiagen, Hilden, Germany) and cloned using the TOPO TA cloning kit for sequencing (Invitrogen, Carlsbad, CA). Four to five clones were sequenced for each $C$. mesnili sample using BigDye v1.1 sequencing mix, and the sequencing reactions were resolved on an ABI 3730 capillary sequencer (Applied Biosystems Inc, Foster City, CA).

Based on the sequence alignment with representative rDNA sequences from GenBank, primers specific to the SSU and ITS

Table 2. Primer sequences used to amplify the small subunit (SSU) and ITS rDNA regions of Caullerya mesnili.

\begin{tabular}{|c|c|c|c|c|c|c|}
\hline Primer name & Species & Primer sequence $\left(5^{\prime}-3^{\prime}\right)$ & rDNA region & Product length & $T^{\mathrm{a}}$ & Source \\
\hline $18 \mathrm{~S} \mathrm{~A}$ & Universal & $\begin{array}{l}\text { CCGAATTCGTCGACAACCTG } \\
\text { GTTGATCCTGCCAGT }\end{array}$ & SSU & $1,630 \mathrm{bp}$ & $54^{\circ} \mathrm{C}$ & Medlin et al. 1988 \\
\hline $18 \mathrm{~S} \mathrm{~B}$ & & $\begin{array}{l}\text { CCCGGGATCCAAGCTTGATCC } \\
\text { TTCTGCAGGTTCACCTAC }\end{array}$ & & & & \\
\hline 18S Cm58 For & Caullerya mesnili & 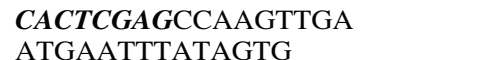 & SSU & $1,568 \mathrm{bp}$ & $52^{\circ} \mathrm{C}$ & This study \\
\hline $18 \mathrm{~S} \mathrm{Cm} 2252 \mathrm{Rev}$ & & $\begin{array}{l}\text { CAGGATCCCCTCTAAA } \\
\text { TCATTTCATTCGAT }\end{array}$ & & & & \\
\hline $\begin{array}{l}18 \mathrm{~S} \mathrm{Cm} 1469 \text { For } \\
28 \mathrm{~S} \mathrm{Cm} 1-1 \mathrm{Rev}^{\mathrm{b}} \\
28 \mathrm{~S} \mathrm{Cm} 1-2 \mathrm{Rev}^{\mathrm{b}}\end{array}$ & Caullerya mesnili & $\begin{array}{l}\text { AGCACAAGTCCTTAACTTGTGTT } \\
\text { CACTCGCCGTTACTGAGGGAATC } \\
\text { CATTCGCCATTACTAAGGGAATC }\end{array}$ & ITS & $621 \mathrm{bp}$ & $54^{\circ} \mathrm{C}$ & This study \\
\hline
\end{tabular}

${ }^{\mathrm{a}}$ Annealing temperature of the respective primer pair.

${ }^{\mathrm{b}}$ Both reverse primers were used in a $1: 1$ ratio for PCR.

Linker regions containing restriction enzyme recognition sites are typed in bold italic.

$\mathrm{PCR}$, polymerase chain reaction. 
regions of $C$. mesnili were designed to avoid amplification of Daphnia rDNA (see Table 2 for all primer information). Polymerase chain reaction with parasite-specific primers was carried out as described above and most SSU rDNA amplicons were sequenced directly. For all ITS regions and some SSU regions, direct sequencing was not possible due to insertions/deletions in one of the many rDNA copies. Therefore, amplicons of the SSU rDNA were cloned with the TOPO TA cloning kit (Invitrogen), whereas amplicons of the ITS region were cloned with the CloneJET PCR cloning kit (Fermentas $\mathrm{GmbH}$ ), according to the manufacturer's recommendations. Six clones of each parasite sample were sequenced as described above. Nucleotide sequences determined in this study were deposited in the GenBank database (for accession numbers see Table 1).

Phylogeny construction. Based on similarity searches in the NCBI nucleotide database with our $C$. mesnili SSU rDNA sequence, we created a list of sequences with which to place the parasite into the eukaryote phylogeny. We included many sequences from members of the class Ichthyosporea, as sequences from this clade were the closest matches in the BLAST search. We used members of the choanoflagellates, fungi, and animals to provide a range of outgroup taxa. The sequences were aligned using CLUSTALX 1.8 (Thompson, Higgins, and Gibson 1994) and edited in BioEdit 7.09 (Hall 1999). All gaps and regions of ambiguous alignment were excluded from the analysis. We tested for the best-fit model of sequence evolution using MODELTEST (Posada and Crandall 1998). The model selected was the general time-reversible model, with invariable sites and rate variation among sites. PHYLIP 3.6 was used for the maximum-likelihood analysis with the programs SEQBOOT, DNAML, and CONSENSE
(Felsenstein 1989). We used 1,000 likelihood replicates, random order addition, global optimization, and 10 jumbles. Bayesian analysis was done with MrBayes 3.2 (Huelsenbeck and Ronquist 2001), using two runs with three heated and one cold chain each. The starting trees were generated randomly. One million generations were run with a 1/100 sample frequency and a burn-in period of 2,500 trees. An average standard deviation of the split frequencies below 0.02 was used to determine convergence.

Polymorphism in the SSU rDNA region. All cloned parasite sequences were analyzed for their percentage of single nucleotide polymorphisms (SNPs), including insertions/deletions (indels). Indels larger than $1 \mathrm{bp}$ were counted as single SNPs, as they could have resulted from a single event.

Morphology and ultrastructure. Ten infected Daphnia (with the parasite strain isolated from Greifensee, see Table 1) and 10 control Daphnia of the same D. galeata genotype were fixed in $4 \%(\mathrm{v} / \mathrm{v})$ glutaraldehyde in Sorensen's phosphate buffer $(0.03 \mathrm{M}$ $\mathrm{KH}_{2} \mathrm{PO}_{4}$ and $0.12 \mathrm{M} \mathrm{Na}_{2} \mathrm{HPO}_{4}$ ) and afterwards washed 5 times with the same buffer for a total of $5 \mathrm{~min}$. Post-fixation was carried out with $1 \%(\mathrm{w} / \mathrm{v}) \mathrm{OsO}_{4}$ in the same buffer. After fixation the animals were again washed in Sorensen's phosphate buffer, dehydrated in graded acetone solutions, and finally embedded in the epoxydic resin EPON. Transversal sections were made through regions of the hepatic caeca, upper intestine, and lower intestine. For the histological sections used in light microscopy, semi-thin sections were prepared $(0.7-1 \mu \mathrm{m})$. Staining of both host and parasite tissue was carried out using Richardson's dye (Richardson, Jarrett and Finke 1960). For visualization of structures using electron microscopy, we cut ultrathin sections $(60 \mathrm{~nm})$, followed by staining with uranyl acetate and lead citrate.

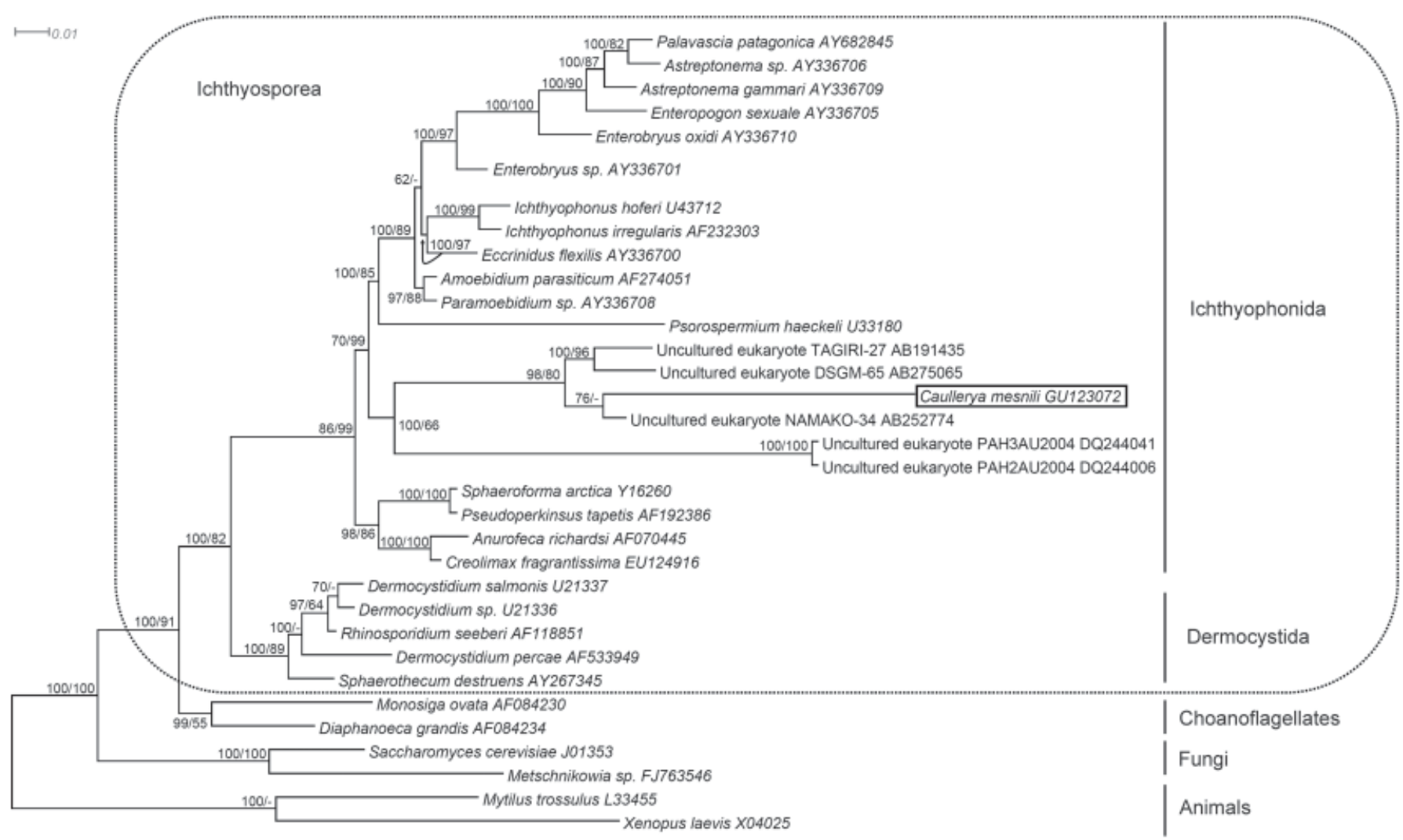

Fig. 1. Phylogenetic comparison of small subunit rDNA sequences from Caullerya mesnili and 26 members of the Ichthyosporea, showing that C. mesnili is a member of the ichthyosporean clade. Numbers at nodes represent Bayesian posterior probabilities and maximum likelihood bootstrap values over $50 \%$. Accession numbers follow the names of each sequence. 


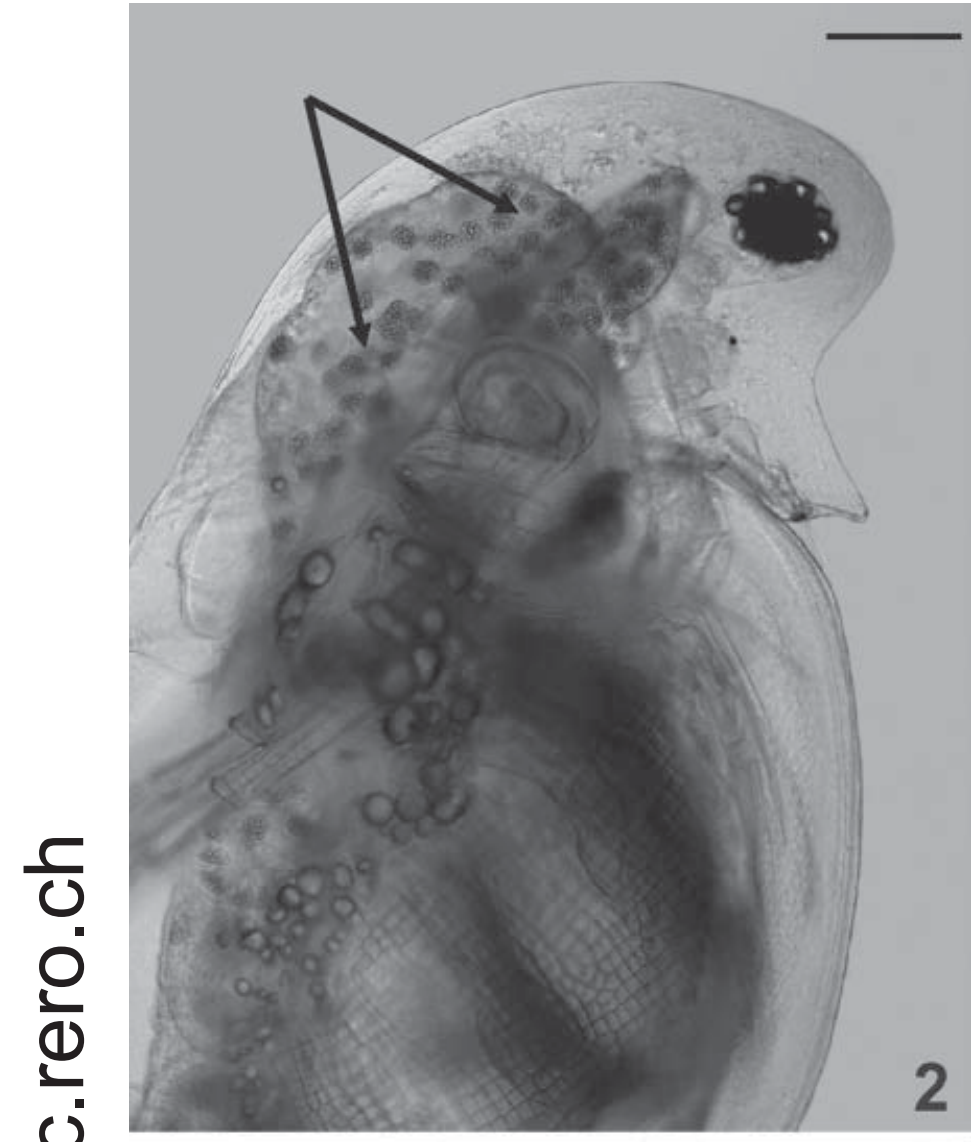

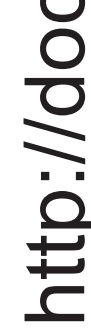

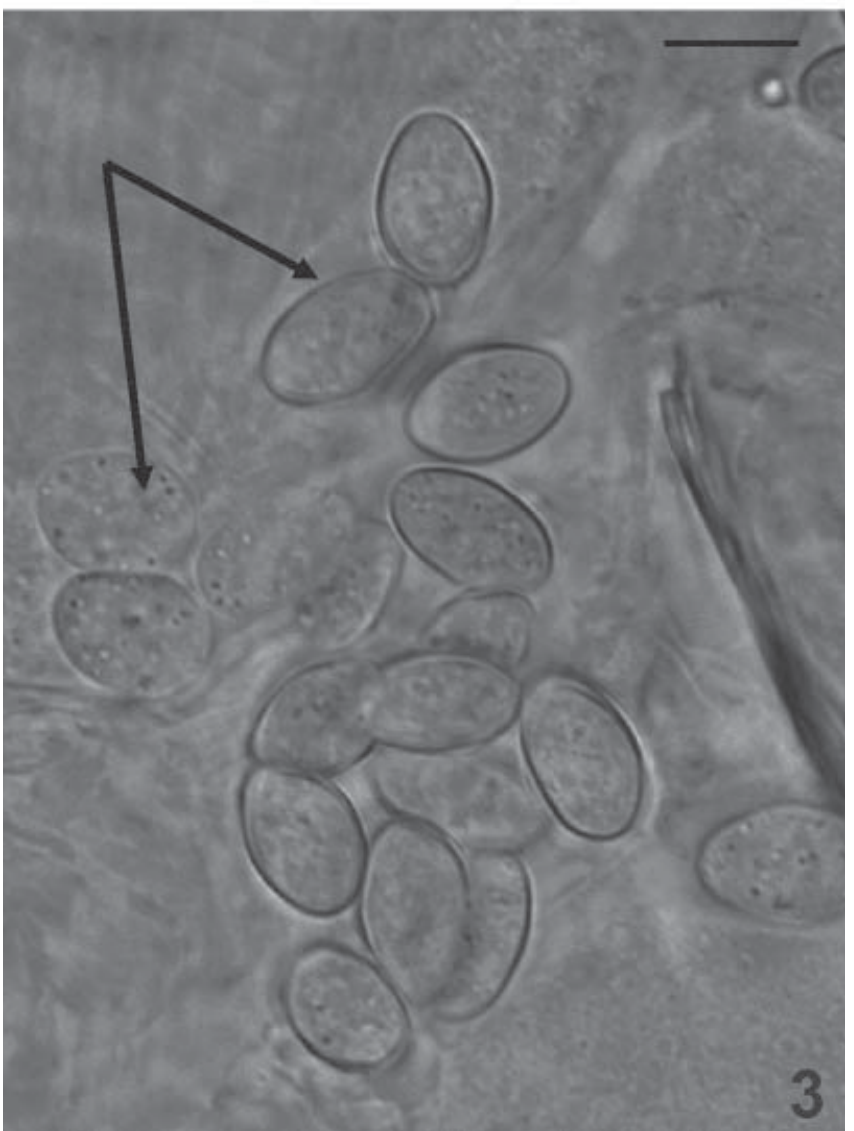

Experiments addressing the virulence, development, and transmission of Caullerya mesnili. The following cultivating conditions were applied for two generations before the experiments to reduce maternal effects on the D. galeata hosts, and in all experimental surveys, if not stated otherwise: artificial medium as above was changed every second day; $20^{\circ} \mathrm{C} ; 16: 8$ light-dark cycle; daily diet of batch-cultured $S$. obliquus of final concentration $1 \mathrm{mg}$ carbon/L. All experiments were started with 2-3day-old juvenile Daphnia, all of a single genotype (isolated from Greifensee).

Parasite virulence and development. Fifty juvenile D. galeata were placed individually per jar in $5 \mathrm{ml}$ of artificial medium. Fifty infected adult $D$. galeata were ground up and distributed equally among these experimental jars. An additional 30 control D. galeata were established under the conditions described above, except ground-up uninfected $D$. galeata were added to each jar. For the next 6 days the jars were stirred twice per day, by gently pipetting the medium up and down to resuspend spores and increase their encounter rates with neonates. The medium was not changed during the infection period (i.e. Days 0-6 post-infection). On Days 3 and 4 post-infection, $5 \mathrm{ml}$ of fresh medium were added to the jars. Starting from Day 8 and every second day thereafter, each $D$. galeata individual, including controls, was observed for infection at $250 \mathrm{X}$ magnification using a stereomicroscope (Zeiss, Göttingen, Germany). The number and location of spore clusters in the intestine (i.e. hepatic caeca, upper intestine, and lower intestine) were recorded. At Day 17 post-infection all surviving individuals were measured for body size. The infected animals and controls were compared for body size ( $t$-test), spore location (ANOVA), as well as for the total number of broods, total number of offspring, age at first reproduction, and time to host death (Kruskal-Wallis). The experiment was terminated when all infected individuals had died.

Identification of transmission stage. Five heavily infected and five control $D$. galeata were selected from lab cultures, washed in distilled water to remove as many microorganisms as possible, and placed individually on depression slides. The fecal material was first examined for the presence of spores and then placed in a moist chamber for 7 days. The slides were observed twice each day for any further development of spores. The above procedure was repeated with the addition of five ground-up uninfected $D$. galeata per slide to determine if exposure to host tissues would induce spore hatching. We attempted to hatch spores as the subsequent release of an amoeboid or flagellate stage is a common character in several species of ichthyosporean (Mendoza et al 2002).

\section{RESULTS}

\section{Molecular data.}

Phylogeny construction. The SSU rDNA sequence of $C$. mesnili was aligned with taxa of the class Ichthyosporea, including several closely matching uncultured eukaryotes and outgroup taxa. The resulting alignment was $1,287 \mathrm{bp}$ long. The class Ichthyosporea formed a monophyletic group (100/82; Bayesian posterior probability/maximum likelihood bootstrap values; Fig. 1), as did the order Ichthyophonida (86/99) and the order Dermocystida (100/89). Caullerya mesnili nested within the Ichthyophonida, but was distinct from all identified species, clus-

Fig. 2-3. Light microscopy of Caullerya mesnili. 2. Daphnia galeata infected with $C$. mesnili, arrows point to spore clusters in the gut epithelium, scale bar $=80 \mu \mathrm{m}$. 3. Spores isolated from dissected spore clusters, arrows indicate individual spores, scale bar $=8 \mu \mathrm{m}$. Photos by Petr Jan Juračka. 


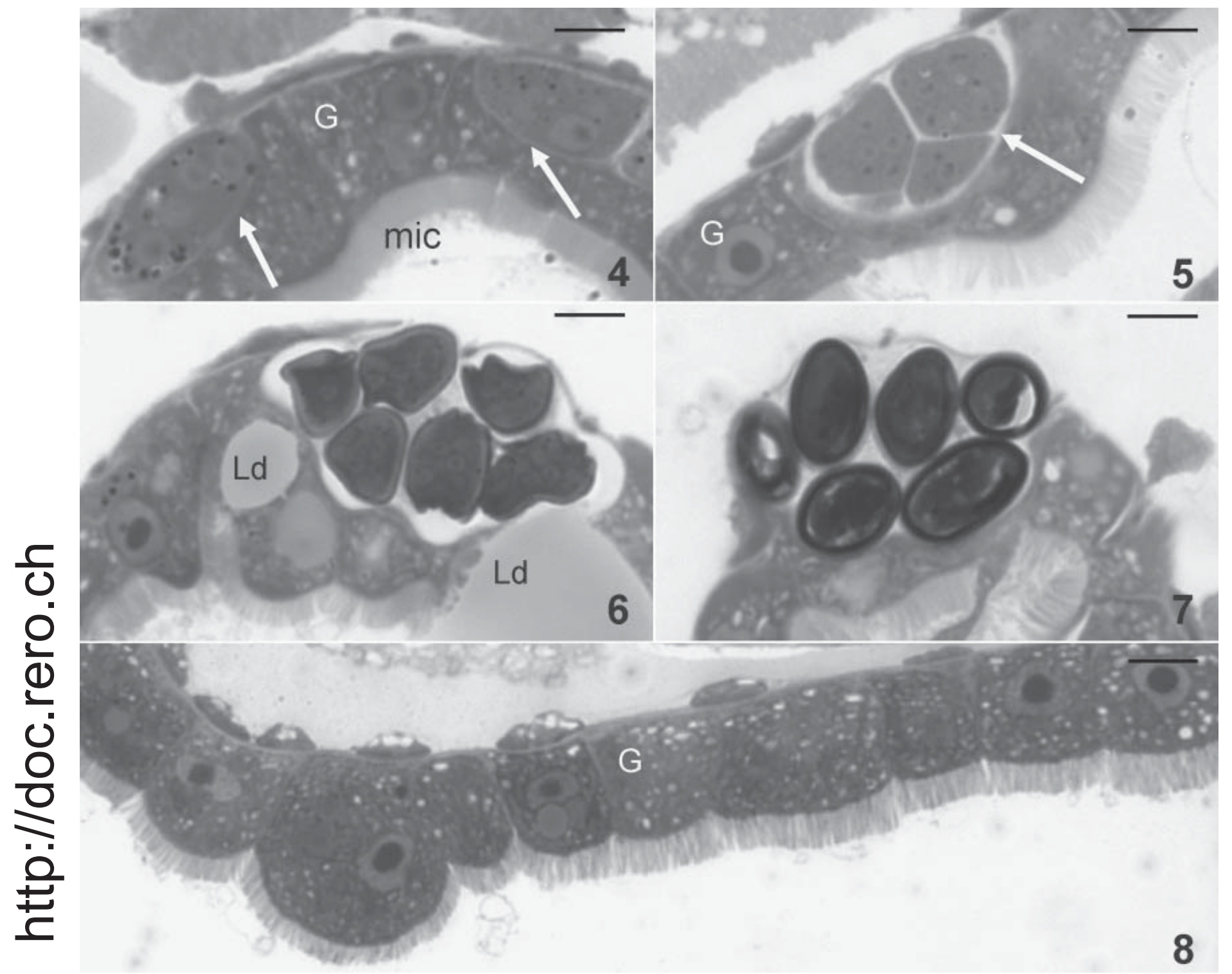

Fig. 4-8. Histological sections showing four developmental stages of Caullerya mesnili in the gut of Daphnia galeata. 4. Juvenile stage (white arrows) surrounded by host gut cells (G) with microvilli (mic) visible along the gut lining, scale bar $=5.5 \mu \mathrm{m}$. 5. Intermediate stage (white arrow), scale bar $=4 \mu \mathrm{m}$. 6. Star stage, the cell wall is thicker, gray inclusions are lipid droplets (Ld) produced by Daphnia, scale bar $=6 \mu \mathrm{m}$. 7. Final stage, a thick spore wall is visible and the spores are ellipsoid, scale bar $=5 \mu \mathrm{m}$. 8. Control section through the anterior intestine of a healthy $D$. galeata, scale $\mathrm{bar}=5 \mu \mathrm{m}$

tering instead with five uncultured eukaryotes (100/66). However, C. mesnili was quiet divergent within this grouping, sharing just $77 \%$ sequence identity with its closest match NAMAKO-34, whereas the other environmental isolates were more closely related to each other (e.g. 94\% identity between NAMAKO-34 and TAGIRI-27). The ITS region was not informative for phylogenetic analysis, as there are fewer ITS sequences for ichthyosporeans in GenBank. Moreover, the C. mesnili ITS sequence showed high within-host polymorphism and was considerably different from any published ITS sequence.

Polymorphism in the SSU rDNA regions. Within-host nucleotide polymorphism in the SSU rDNA and ITS regions was observed for all cloned parasite sequences. Differences between clonal variants were mostly characterized by single base-pair substitutions, whereas nucleotide insertions and deletions (indels) were rare and short (i.e. $1-6 \mathrm{bp}$ ). The 10 cloned isolates of C. mesnili differed in the amount of detected polymorphism in the ITS region. For example, the sequence from Ammersee represented the lowest sequence divergence $(1.02 \%$ polymorphic sites), whereas the sequence from the Seč reservoir showed the highest sequence divergence ( $4.43 \%$ polymorphic sites). As expected, polymorphism in the SSU rDNA was lower than in the ITS region $[0.75 \pm 0.03 \%$ (SE) compared with $2.27 \pm 0.36 \%$ polymorphic sites, respectively]. Theoretically, Taq DNA polymerase errors and/or cloning artifacts may contribute to the observed level of within-host parasite sequence variation. We tested for this error in a previous study of a microsporidian parasite by subjecting the parasite clones to a second round of PCR and cloning. We found the misincorporation of nucleotides by the same Taq DNA polymerase to be 100 -fold lower $\left(1.3 \times 10^{-4}\right.$ errors per 
site) than the sequence polymorphism detected and therefore negligible (Wolinska, Giessler and Koerner 2009).

Morphology and ultrastructure. Caullerya mesnili infections are clearly visible in infected animals (Fig. 2), as spore clusters throughout the intestine (Fig. 3). Analysis of sections through the intestinal tract of infected daphniids revealed the presence of spores in what appear to be four major developmental stages. The first stage of infection (called here the juvenile stage) is irregularly shaped and multinucleate, located along the base of the intestinal lining (Fig. 4). A second stage (called here the intermediate stage), is characterized by cell division, accompanied by the partitioning of multiple nuclei between the forming spores (Fig. 5). In a third stage (called here the star stage as in cross-section the spores appear star-like) cell division seems complete and the multinucleate cells, which are now spore-like in appearance, have a thickened wall (Fig. 6). In a final stage the cells are ovoid with thick spore walls (Fig. 7). The gut epithelium of uninfected daphniids did not contain any parasite features (Fig. 8). Several sections showed what appear to be final-stage spore clusters breaking through the gut epithelium (Fig. 9) and single spores were often seen in the gut lumen (Fig. 10).

Electron micrographs confirmed that $C$. mesnili is an intercellular parasite, taking up residence along the basal lamina of Daphnia's gut epithelium. The cytoplasm of spores has a granular appearance (Fig. 11), which is seen in the juvenile, intermediate and star stages. We could not definitively identify the granular structures. However, they are similar in appearance to ribosomes as well as to glycogen. Juvenile spores contain multiple nuclei and have a highly irregular and convoluted plasma membrane (Fig. 11). In the star stage the spores are again pleurinucle-

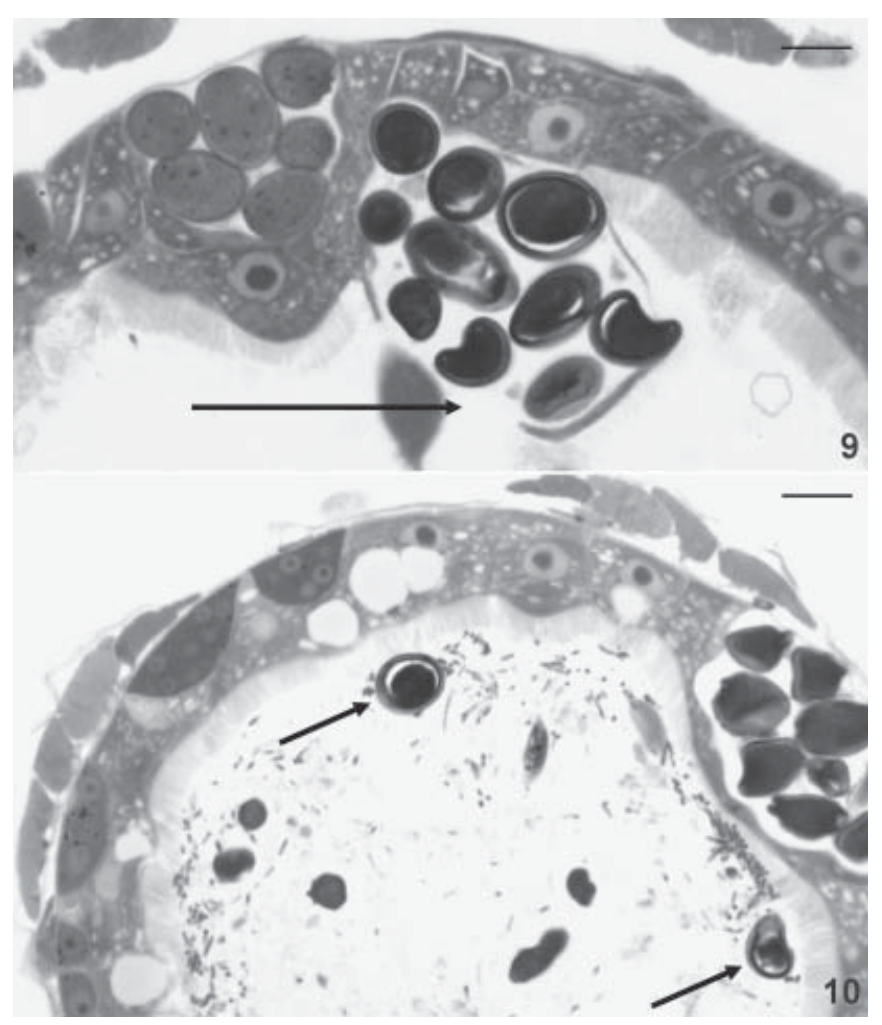

Fig. 9-10. Spore cluster of Caullerya mesnili in the gut epithelium of Daphnia galeata. 9. Spore cluster breaking through the gut epithelium (black arrow), scale bar $=7 \mu \mathrm{m}$. 10. Individual spores in the gut lumen (black arrows), scale bar $=10 \mu \mathrm{m}$. ate and individual spores are often at varying degrees of maturity (i.e. some have thicker spore walls or are more oval shaped than others). Further, as in the light microscopy, a thick cell wall is clearly visible (Fig. 12). Unfortunately, the fixative was not able to penetrate the thick spore wall of the mature spores. As a result, we are not able to describe its inner structures. However, the spore wall and its features were well preserved, showing a distinct layering pattern, with several thinner inner layers and a thicker outer layer (Fig. 12).

Experiments addressing the virulence, development, and transmission of Caullerya mesnili

Parasite virulence. Twenty-five of the 50 exposed D. galeata became infected with $C$. mesnili. Only those Daphnia that became infected were analyzed for differences from the controls. Fecundity, body size, and lifespan of infected animals were significantly reduced. Infected animals produced one or two small clutches (i.e. average number of clutches from infected animals: $1.7 \pm 0.14$, control animals: $\left.5.2 \pm 0.25 ; H_{1,37}=29.3, P<0.001\right)$ after which reproduction stopped (i.e. average number of offspring from infected animals: $6.0 \pm 0.60$, control animals: $\left.28.5 \pm 1.14 ; H_{1,37}=27.6, P<0.001\right)$. In addition, age at first reproduction was increased in infected animals, which produced their first clutch at day $7.1 \pm 0.44$ post-infection, whereas control animals produced their first clutch at day $5.7 \pm 0.23\left(H_{1,46}=5.86\right.$, $P=0.015$ ). Body size was measured for infected and control animals at Day 17 post-exposure: infected animals were on average $3.65 \%$ smaller than the controls $\left(t_{1,33}=4.3, P<0.001\right)$. Infected Daphnia lived on average $19.2 \pm 0.70$ days post-exposure, whereas most control animals lived until the experiment was terminated at Day $33\left(H_{1,41}=32.0, P<0.001\right)$.

Parasite development within the host. Spore clusters were first visible in the gut epithelium of hosts at Day 9 post-exposure. The first clusters always appeared in the hepatic caeca or in the very upper portion of the intestine. The number of spore clusters increased rapidly from the time when infections were first visible at Day 9 post-infection until Day 13 post-infection (Fig. 13). From Day 13 post-infection until host death, the number of spore clusters in the host gut leveled off with considerable fluctuations. The largest numbers of spores were located in the upper intestine and the hepatic caeca, with few in the lower intestine $\left(F_{2,74}=70.7\right.$, $P<0.001)$.

Parasite transmission. Incubation of isolated spores in a moist chamber for 1 week showed that no further development of the spores occurred under these conditions. Furthermore, hatching was not stimulated by the addition of ground-up D. galeata.

\section{DISCUSSION}

Phylogenetic inference. Caullerya mesnili clustered within the order Ichthyophonida, in a group consisting of a variety of uncultured eukaryotes from both marine and brackish waters. The fact that within a small branch on the ichthyosporean tree there are species from vastly different geographical locations (i.e. Japan, Europe, and the Atlantic ocean) and habitats (i.e. marine thermal vents, saline lakes, and freshwater lakes) supports the idea that there remains a large amount of undocumented biodiversity in the Ichthyosporea, similar to that for many other groups of microbial eukaryotes (Moreira and Lopez-Garcia 2003; Takishita et al. 2005). Specifically, C. mesnili shared maximum identity with the uncultured eukaryote NAMAKO-34, which was retrieved from the anoxic sediment of the saline and meromictic Lake Namako in Japan (Takishita et al. 2007). The other uncultured eukaryotes that $C$. mesnili clustered with were found in the anoxic sediment around fumaroles on the ocean floor (Takishita et al. 2005), or were collected from the water column of the meromictic Lake Pavin in France (Lefèvre et al. 2007). Unfortunately, all 


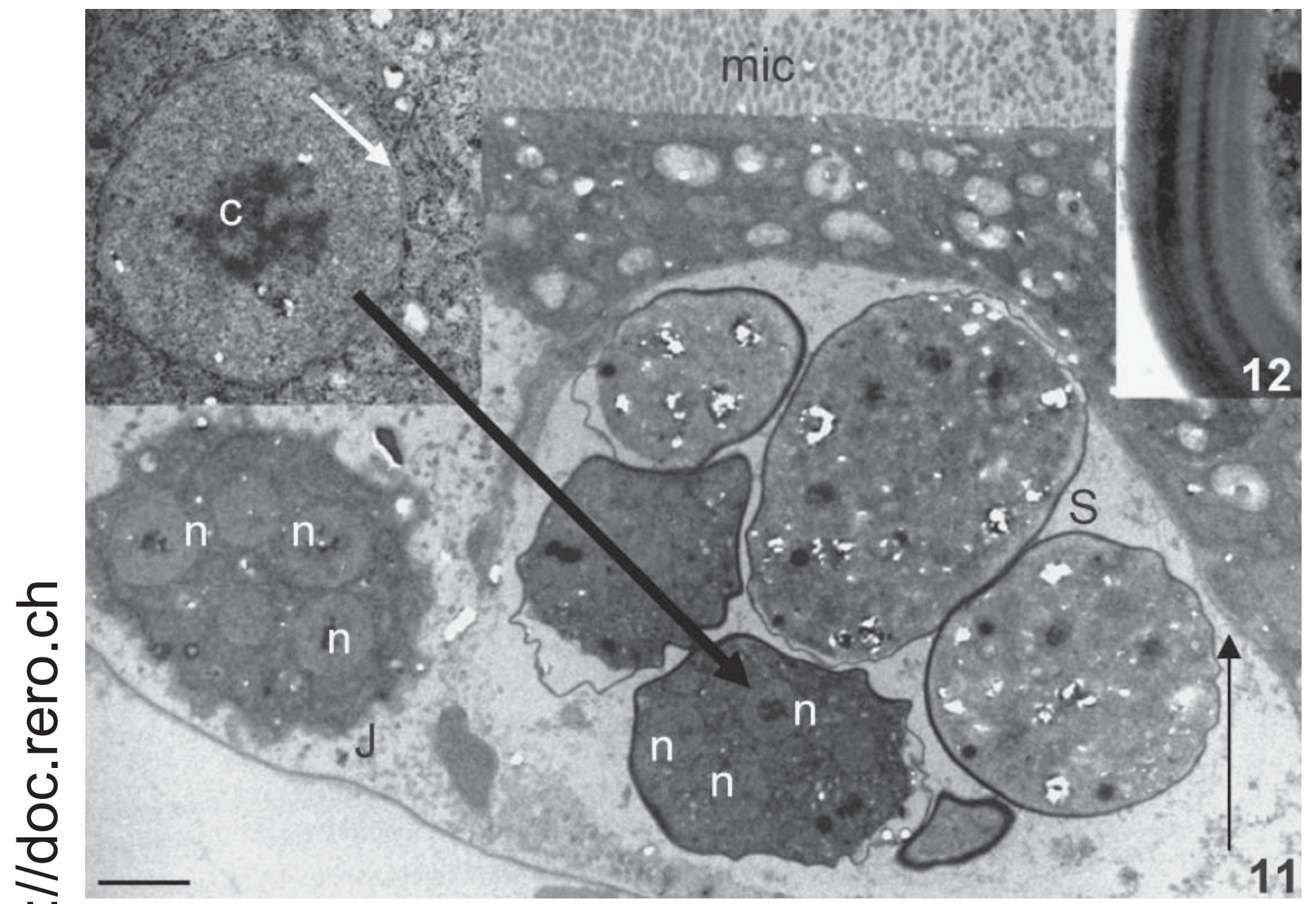

Fig. 11-12. Electron micrographs of Caullerya mesnili within the intestine of Daphnia galeata. 11. The juvenile stage (J) has a highly irregular plasma membrane and multiple nuclei (n). The star stage (S) also contains multiple nuclei (n), with spores at various degrees of maturity. The demarcation between host and parasite tissue is marked by the thin black arrow. At the top of the picture, host microvilli (mic) are visible, protruding from intestinal cells. A detailed view of a nucleus shows the nuclear membrane (white arrow) and condensed chromatin (c, left inlay). Scale bar $=2 \mu \mathrm{m} .12$. Final-stage spores have a thick spore wall with visible layering.

these closest relatives of $C$. mesnili are unknown species, identified only from environmental samples. Therefore, we cannot identify any unique or common characters for this grouping.

The other relationships in the ichthyosporean phylogeny were the same as those published previously (e.g. Cafaro 2005; Marshall et al. 2008). The Ichthyophonida formed four clades, which are well supported by the bootstrap values and posterior probabilities. The largest group contained members of the Eccrinales, until recently grouped with the fungi (see Cafaro 2005), as well as the genus Ichthyophonus and two Amoebidiales - Paramoebidium sp. and Amoebidium parasiticum. Psorospermium haeckeli on the other hand constituted its own group (as in Marshall et al. 2008), as did all the uncultured eukaryotes that grouped with C. mesnili. Finally, there was a fourth group consisting of Anurofeca richardsi, Creolimax fragrantissima, Pseudoperkinsus tapetis, and Sphaeroforma arctica. The other order of ichthyosporeans, the Dermocystida, formed a clade of the five described species, with Sphaerothecum destruens grouping outside the remaining four members.

Caullerya mesnili and Amoebidium parasiticum were both discovered by Chatton over a century ago. Only now, with the advent of modern sequencing techniques have they been placed within the Ichthyosporea. The situation is similar for many ichthosporeans, such as the Eccrinales, which were considered fungi until 2005 (Cafaro 2005). Thus, there likely remain many misclassified ichthyosporeans. The future reclassification of these species will add to the knowledge and diversity of the Ichthyosporea.

Polymorphism in the rDNA region. Cloning of the rDNA region from several $C$. mesnili isolates revealed within-host sequence variation, especially in the ITS region. This indicates that units of the rDNA multigene family comprise multiple genetic variants (i.e. intragenomic variation), which is common across various species (e.g. Harris and Crandall 2000; Parkin and Butlin 2004; Whang et al. 2002). Alternatively, Daphnia may have been infected by multiple parasite strains. However, the laboratory sequence, which showed a similar amount of variation as the field sequences, has been passed through approximately 90 host generations, considering a host generation time of 10 days (Wolinska, Löffler and Spaak 2007b). Such passage is expected to lower the number of coexisting strains. However, the rDNA sequence still showed genetic polymorphism, supporting the conclusion that its origin is intragenomic. 


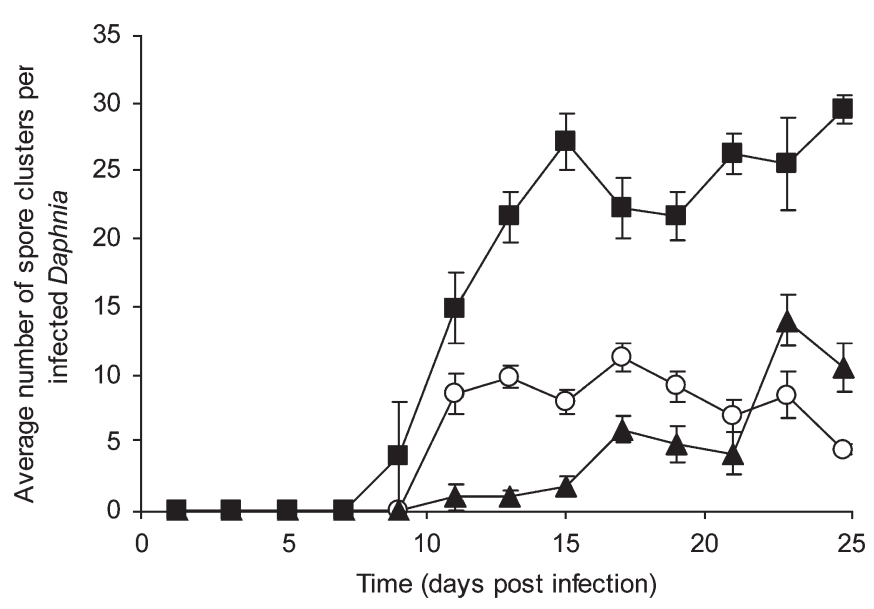

Fig. 13. Average number and location of visible Caullerya mesnili spore clusters in the intestine of Daphnia galeata over the course of infection. Values are mean \pm standard error. The number of replicates decreases over time as infected hosts die. $\bigcirc$, hepatic caeca; $\square$, upper intestine; $\boldsymbol{\Delta}$, lower intestine.

Assignment of Caullerya mesnili to the class Ichthyosporea, order Ichthyophonida. Based on our results, we propose that C. mesnili should be removed from the phylum Haplosporidia and placed in the class Ichthyosporea, specifically in the order Ichthyophonida. Caullerya mesnili lacks any definitive haplosporian characters, whereas the molecular, morphological, and ecological features of this parasite are consistent with members of the class Ichthyosporea. These features include: high sequence similarity, a multinucleate stage, thick cell walls, and spore residence in the gut tissue of an aquatic host.

Development within Daphnia. As further spore development was not observed outside of the host, spores likely serve as the infective stage to daphniids. The spores are probably taken up during grazing, as in other Daphnia parasites (Ebert 2005). How C. mesnili penetrates the intestinal epithelium remains unknown. However, once inside the host $C$. mesnili establishes itself intercellularly, taking up residence in the extracellular matrix along the basal lamina. The parasite then continues its development, undergoing cell division, the secretion of a thick cell wall, and a change in conformation, becoming ovoid. It is probably no matter of chance that most spore clusters are located in the hepatic caeca and upper intestine, this being the major site of nutrient absorption in the Daphnia gut (Hardy and MacDougall 1895). Once the spores are mature, the membrane surrounding the spore cluster appears to break open releasing individual spores into the lumen of the intestine. Light and electron micrographs showed that the juvenile, intermediate, and star stages are pleurinucleate, in accordance with the description of $C$. mesnili by Green (1974). Unfortunately, due to the incomplete fixation of the final-stage spores, we cannot be certain if this stage is also pleurinucleate.

The majority of ichthyosporeans infect via a uni- or bi-nucleate stage, which is often released from a multinucleate structure (Mendoza et al. 2002). Thus, a multinucleate infective stage seems unlikely for $C$. mesnili. Future research on the life cycle of C. mesnili should continue to investigate spore hatching via the establishment of an in vitro culture, as this is key to understanding the infection process. Furthermore, the use of in situ hybridization will help to map how infections are established and spread throughout host tissue.

Caullerya mesnili spreads rapidly throughout the host gut; but the mechanism remains unclear. The simplest means would be the break-up of juvenile spore clusters for propagation. However, we found no direct evidence to support this hypothesis in the histological sections (i.e. the separation of juvenile spore clusters). Alternatively, there may be a second spore type that is responsible for the spread of infections throughout the host. Members of another ichthyosporean group, the Eccrinales, have such a system (Cafaro 2005). Uni-nucleate spores (oval to ellipsoidal in shape and with thick walls) serve as the transmission stage to new hosts, whereas multinucleate spores (more elongated in shape with thin walls) function for germination within the same host, thus spreading the disease (Cafaro 2005). It is possible that some C. mesnili spore clusters develop into germination spore types.

Host-parasite interactions. Parasites can play a regulatory function in host populations when the virulence of the parasite decreases the net growth rate of its host (Anderson and May 1981). Previous work established that $C$. mesnili decreases fecundity, growth, and life-span of its hosts (Bittner et al. 2002; Wolinska et al. 2006), which we confirmed in the present study. Transmission likely occurs when naive hosts ingest spores during grazing. The thick spore wall suggests that spores may persists for extended periods of time as do spores of other Daphnia parasites (see Ebert 2005), as well as other ichthyosporeans (Cafaro 2005).

The reclassification of $C$. mesnili brings a new wealth of ecological data to the Ichthyosporea. Although we were unable to describe the complete life cycle, we have described some developmental stages and their corresponding morphology. In general ichthyosporean life cycles are complex and poorly understood. Documentation of the entire life cycle of C. mesnili requires further study, and will be aided immensely by the use of in situ hybridization, now that sequence data are available.

\section{ACKNOWLEDGMENTS}

We would like to thank M. Kredler and R. Jaenichen for assistance in the lab and C. Schoebel for providing the $C$. mesnili used in the experimental work. Thank you to B. Keller, C. Schoebel, and C. Tellenbach for conducting some preliminary molecular work with this parasite. We also thank A. Petrusek and J. Seda for providing infected Daphnia from their field study of Czech reservoirs. Many thanks to two anonymous reviewers for their valuable comments and suggestions. This research was funded by a DFG grant (WO 1587/2-1), a grant from the Volkswagen Stiftung through the EES-LMU program, and by departmental research funds provided by Prof. Wilfried Gabriel.

\section{LITERATURE CITED}

Anderson, R. M. \& May, R. M. 1981. The population dynamics of microparasites and their invertebrate hosts. Phil. Trans. R. Soc. Lond., 291:451-524.

Bittner, K., Ebert, D. \& Rothhaupt, K.-O. 2002. Ecological interactions of the microparasite Caullerya mesnili and its host Daphnia galeata. Limnol. Oceanogr., 47:300-305.

Cafaro, M. J. 2005. Eccrinales (Trichomycetes) are not fungi, but a clade of protists at the early divergence of animals and fungi. Mol. Phylogenet. Evol., 35:21-34.

Cavalier-Smith, T. 1998. Neomonada and the origin of animals and fungi. In: Coombs, G., Vickerman, K., Sleigh, M. \& Warren, A. (ed.), Evolutionary Relationships among Protozoa. Chapman \& Hall, London. p. 375-407.

Chatton, E. 1907. Caullerya mesnili n. g. n. sp. Haplosporidie parasite des Daphnies. Soc. Biol., 62:529-531.

Ebert, D. 2005. Ecology, Epidemiology, and Evolution of Parasitism in Daphnia [Internet]. National Library of Medicine (US), National Center for Biotechnology Information, Bethesda, MD. Available at http:// www.ncbi.nlm.nih.gov/entrez/query.fcgi?db=Books

Felsenstein, J. 1989. PHYLIP-Phylogeny inference package. Cladistics, 5:164-166. 
Green, J. 1974. Parasites and epibionts of Cladocera. Phil. Trans. R. Soc. Lond. B, 32:417-515.

Hall, T. A. 1999. BioEdit: a user-friendly biological sequence alignment editor and analysis program for Windows 95/98/NT. Nucl. Acids Symp. Ser., 41:95-98.

Hardy, W. B. \& MacDougall, W. 1895. On the structure and function of the alimentary canal of Daphnia. Proc. Camb. Phil. Sot. Boil. Sri., 8:41-50.

Harris, D. J. \& Crandall, K. A. 2000. Intragenomic variation within ITS1 and ITS2 of freshwater crayshes (Decapoda: Cambaridae): implications for phylogenetic and microsatellite studies. Mol. Biol. Evol., 17:284-291.

Huelsenbeck, J. P. \& Ronquist, F. 2001. MRBAYES: Bayesian inference of phylogenetic trees. Bioinformatics, 17:754-755.

Jeschke, M. J. \& Tollrian, R. 2000. Density-dependent effects of prey defences. Oecologia, 123:391-396.

Keller, B., Wolinska, J., Tellenbach, C. \& Spaak, P. 2007. Reproductive isolation keeps hybridizing Daphnia species distinct. Limnol. Oceanogr., 52:984-991.

Lass, S. \& Bittner, K. 2002. Facing multiple enemies: parasitized hosts respond to predator karimones. Oecologia, 132:344-349.

Lefèvre, E., Bardot, C., Noel, C., Carrias, J-F., Viscogliosi, E., Amblard, C. \& Sime-Ngando, T. 2007. Unveiling fungal zooflagellates as members of freshwater picoeukaryotes: evidence from a molecular diversity study in a deep meromictic lake. Environ. Microbiol., 9:61-71.

Little, T. J. \& Ebert, D. 2001. Temporal patterns of genetic variation for resistance and infectivity in a Daphnia-microparasite system. Evolution, 55:1146-1152.

Little, T. J. \& Ebert, D. 2004. Evolutionary dynamics in Daphnia and their microparasites. In: Dronamraju, K. R. (ed.), Infectious Disease and Host. Cambridge University Press, Cambridge, UK. p. 222-240.

Marshall, W. L., Celio, G., McLaughlin, D. J. \& Berbee, M. L. 2008. Multiple isolations of a culturable, motile Ichthyosporean (Mesomycetozoa, Opisthokonta), Creolimax fragrantissima n. gen., n. sp. from marine invertebrate digestive tracts. Protist, 159:415-433.

Medlin, L., Elwood, H. J., Stickel, S. \& Sogin, M. L. 1988. The charac terization of enzymatically amplified eukaryotic 16s-like rRNA-coding regions. Gene, 71:491-499.

Mendoza, L., Taylor, J. W. \& Ajello, L. 2002. The class Mesomycetozoa: a heterogeneous group of microorganisms at the animal-fungal boundary. Апnи. Rev. Microbiol., 56:315-344.

Moreira, D. \& Lopez-Garcia, P. 2003. Are hydrothermal vents oases for parasititc protists? Trends Parasitol., 19:556-558.

Parkin, E. J. \& Butlin, R. K. 2004. Within- and between-individual sequence variation among ITS1 copies in the meadow grasshopper Chorthippus parallelus indicates frequent intrachromosomal gene conversion. Mol. Biol. Evol., 21:1595-1601.

Petrusek, A., Hobæk, A., Nilssen, J. P., Skage, M., Černý, M., Brede, N. \& Schwenk, K. 2008. A taxonomic reappraisal of the European Daphnia longispina complex (Crustacea, Cladocera, Anomopoda). Zool. Scripta, 37:507-519.

Posada, D. \& Crandall, K. A. 1998. MODELTEST: testing the model of DNA substitution. Bioinformatics, 14:817-818.

Ragan, M. A., Goggin, C. L., Cawthorn, R. J., Cerenius, L., Jamieson, A. C., Plourde, S. M., Rand, T. G., Söderhäll, K. \& Gutell, R. R. 1996. A novel clade of protozoan parasites near the animal-fungal divergence. Proc. Natl. Acad. Sci., 93:11907-11912.

Richardson, K. C., Jarrett, L. \& Finke, E. H. 1960. Embedding in epoxy resins for ultrathin sectioning in electron microscopy. Stain Technol., 35:313-323.

Seda, J., Petrusek, A., Machacek, J. \& Smilauer, P. 2007. Spatial distribution of the Daphnia longispina species complex and other planktonic crustaceans in the heterogeneous environment of canyon-shaped reservoirs. J. Plank. Res., 29:619-628.

Takishita, K., Miyake, H., Kawato, M. \& Maruyama, T. 2005. Genetic diversity of microbial eukaryotes in anoxic sediment around fumaroles on a submarine caldera floor based on the small-subunit rDNA phylogeny. Extremophiles, 9:185-196.

Takishita, K., Tsuchiya, M., Kawato, M., Oguri, K., Kitazato, H. \& Maruyama, T. 2007. Genetic diversity of microbial eukaryotes in anoxic sediment of the saline meromictic Lake Namako-ike (Japan): on the detection of anaerobic or anoxic-tolerant lineages of eukaryotes. Protist, 158:51-64.

Thompson, J. D., Higgins, D. G. \& Gibson, T. J. 1994. CLUSTAL W: improving the sensitivity of progressive multiple sequence alignment through sequence weighting, position-specific gap penalties and weight matrix choice. Nucleic Acids Res., 22:4673-4680.

Whang, I. J., Jung, J., Park, J. K., Min, G. S. \& Kim, W. 2002. Intragenomic length variation of the ribosomal DNA intergenic spacer in a malaria vector, Anopheles sinensis. Mol. Cells, 14:158-162.

Wolinska, J., Bittner, K., Ebert, D. \& Spaak, P. 2006. The coexistence of hybrid and parental Daphnia: the role of parasites. Proc. R. Soc. Lond. $B, 273$ : 1977-1983.

Wolinska, J., Keller, B., Manca, M. \& Spaak, P. 2007a. Parasite survey of a Daphnia hybrid complex: host specificity and environment determine infection. J. Anim. Ecol., 76:191-200.

Wolinska, J., Löffler, A. \& Spaak, P. 2007b. Taxon specific reaction norms to predator cues in a hybrid Daphnia complex. Freshwater Biol., 52:1198-1209.

Wolinska, J., Giessler, S. \& Koerner, H. 2009. Molecular identification and hidden diversity of novel Daphnia parasites from European lakes. Appl. Environ. Microbiol., 75:7051-7059. 